\title{
A Method for QR Code Decryption Based on SMS4
}

\author{
Ye Yuan \\ Department of Computer Science, North China Electric Power University, Baoding 071000, China \\ hong198520@163.com
}

Keywords: SMS4; QR code.

\begin{abstract}
The QR code to pay not only represents people's new way of life, is also one of hot topics in the study of the current electronic payment field. But for the sake of lack of mature and safe payment scheme, being unable to further open up capital chain to complete settlement of funds to guarantee the safety of the payment process, Domestic QR code payment has not been popular, still mainly stays in the information application level such as retrieving goods, serving as a proof of payment. This paper uses the SMS4 algorithm to encrypt plaintext of QR codes, has effectively solved security issues about short key length in DES.
\end{abstract}

\section{Introduction}

With the development of economy and the maturity of the IT technology, the business model paid in $\mathrm{QR}$ code for the media gradually enters into people's horizons. The QR code to pay not only represents people's new way of life, is also one of hot topics in the study of the current electronic payment field. You just need to scan the qr code, which allows the customer in a short period of time to finish all the process from order to pay just through phone. Its convenience has won a wide recognition of industry. But for the sake of lack of mature and safe payment scheme, being unable to further open up capital chain to complete settlement of funds to guarantee the safety of the payment process, Domestic QR code payment has not been popular, still mainly stays in the information application level such as retrieving goods, serving as a proof of payment. Additionally, taking the recent news into consideration, for example, the Central Bank exerted a halt on QR code and using the QR code payment led to being cheated, people are worried about the safety of the qr code payment.

QR codes originated in Japan Denso Company, is a kind of matrix QR code, with large information capacity, high reliability, low cost advantages. QR codes in addition to the English character, also can represent Chinese characters, images and other information medium, widely used in transportation, communications, financial, medical, and service industries. With the widely application of the qr code, its security problem is increasingly serious. The most notable is the customer service center of China railway's train ticket real-name system carried out in recent years. In the beginning, the personal information on the QR code of train ticket entirely use clear storage. Ordinary users can access the personal information only by common sweep code software, which seriously damages citizens' personal privacy. So as QR codes are widely used, its security problem more cannot be ignored.

\section{The existing research}

However, researches of encryption method directing at QR code are not much, and among them Zhang Dinghui in 2011 came up with a theory using DES encryption algorithm to encrypt binary image of QR codes. The method using the classical cryptography algorithm DES as the encryption algorithm, is widely used nowadays. But the encryption speed is slow, and the security of 56 secret key length in the rapid development of computer technology is slightly insufficient. The table 1 shows that even with a brute force cracking, cracking time of DES is only 1 hour. 
Table 1 Exhaustive search key time

\begin{tabular}{ccccc}
\hline Key lengths(bit) & $\begin{array}{c}\text { Encryption } \\
\text { algorithm }\end{array}$ & $\begin{array}{l}\text { Alternative } \\
\text { Number of key }\end{array}$ & $\begin{array}{l}\text { Time taken (encrypt } \\
109 \text { times per second) }\end{array}$ & $\begin{array}{c}\text { Time taken (encrypt } \\
1013 \text { times per second) }\end{array}$ \\
\hline 56 & DES & $2^{56}=7.2^{*} 10^{16}$ & $2^{55} \mathrm{~ns}=1.125$ years & 1 hour \\
128 & AES/SMS4 & $2^{128}=3.4 * 10^{38}$ & $2^{127} \mathrm{~ns}=5.3^{*} 10^{21}$ years & $5.3^{*} 10^{17}$ years \\
168 & 3DES & $2^{168}=3.7 * 10^{50}$ & $2^{167} \mathrm{~ns}=5.8^{*} 10^{33}$ years & $5.8^{*} 10^{29}$ years \\
192 & AES & $2^{192}=6.3^{*} 10^{57}$ & $2^{191} \mathrm{~ns}=9.8^{*} 10^{40}$ years & $9.8^{*} 10^{36}$ years \\
256 & AES & $2^{256}=1.2^{*} 10^{77}$ & $2^{255} \mathrm{~ns}=1.8^{*} 10^{60}$ years & $1.8^{*} 10^{56}$ years \\
\hline
\end{tabular}

\section{The introduction of the dense SMS4 algorithm}

This paper uses the SMS4 algorithm to encrypt plaintext of QR codes.SMS4 algorithm is the same as the DES. It is also a kind of grouping symmetrical encryption algorithm, in 128, for a set, and the secret key length is 128 bits. If you use a brute force cracking, it will take you $5.3^{*} 10^{17}$ years to crack it, which guarantees the safety. Both the encryption algorithm and key expansion algorithm adopt 32 rounds of nonlinear iterative structure. The structure of decryption algorithm and the structure of the encryption algorithm are identical except for the reverse sequence of the use of round key, and the order of decryption keys is the reverse order of encryption keys.

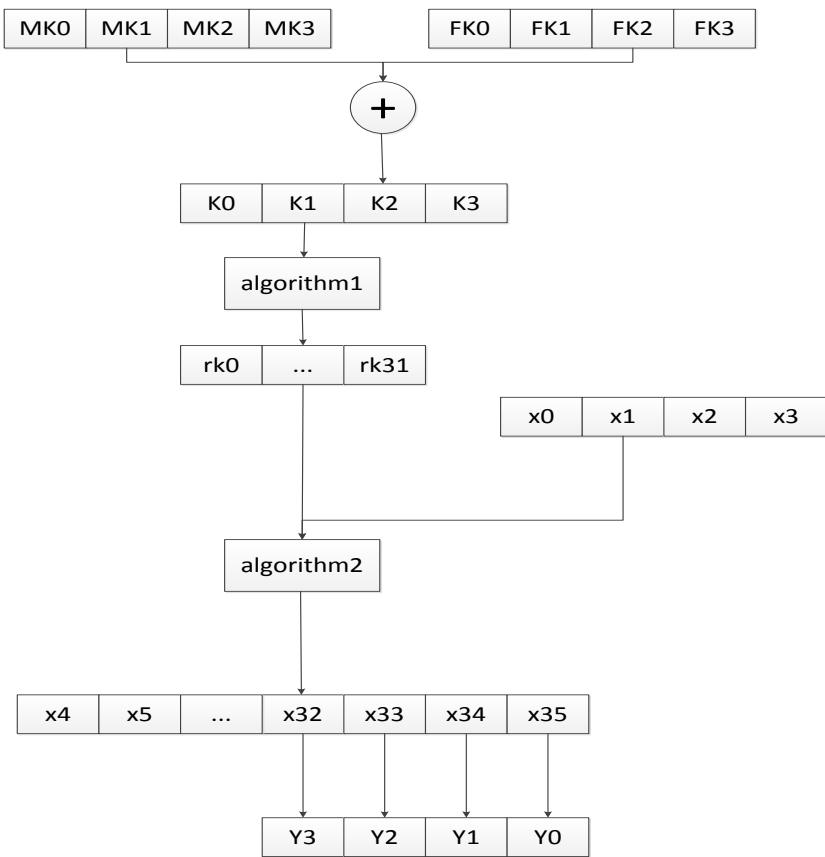

Figure 1 algorithm flow chart

\section{Arithmetic step}

Step 1 , let secret key $M K_{i}(i=0,1,2,3)$ xor the steady index $F K_{i}(i=0,1,2,3)$, we can get the $K_{0}$ to $K_{3}$.

Step 2, use $K_{0}$ to $K_{3}$ to get 32 round secret key $r k_{0}$ to $r k_{31}$ by arithmetic 1 .

Step3, through arithmetic 2 we use the round key and plaintext $X_{0}$ to $X_{3}$ to get $X_{4}$ to $X_{35}$.

Step4, selcet the final four index as the cryptograph $Y_{3}, Y_{2}, Y_{1}, Y_{0}$.

Arithmetic 1 is called round-key-expend arithmetic and we can get round key $r k_{0}$ to $r k_{31}$ by using it. Arithmetic is expressed as follow:

$r k_{i}=K_{i+4}=K_{i} \oplus T^{\prime}\left(K_{i+1} \oplus K_{i+2} \oplus K_{i+3} \oplus C K_{i}\right)$

Arithmetic 2 is the round-function-arithmetic, it's expressed as follow: 


$$
\begin{aligned}
& F\left(X_{0}, X_{1}, X_{2}, X_{3}, r k\right)=X_{0} \oplus T\left(X_{1} \oplus X_{2} \oplus X_{3} \oplus r k\right) \\
& X_{i+4}=F\left(X_{i}, X_{i+1}, X_{i+2}, X_{i+3}, r k_{i}\right)=X_{i} \oplus T\left(X_{i+1} \oplus X_{i+2} \oplus X_{i+3} \oplus r k_{i}\right) \quad(i=0,1, \ldots 31)
\end{aligned}
$$

Through arithmetic2, we will get $\mathrm{x} 4-\mathrm{x} 35$. Finally, let the cryptograph $\left(Y_{3}, Y_{2}, Y_{1}, Y_{0}\right)=$ $\left(X_{35}, X_{34}, X_{33}, X_{32}\right)$.

$T($.$) and T^{\prime}($.$) are replacement synthesis function, can be indicated as follow:$

$T()=.L(\tau()$.

$\tau($.$) is nolinear transformation while L($.$) is linear transformation. \tau($.) will applied reference the Sbox ${ }^{[1]}$. The Sbox is announced by the national bureau of password.

$L($.) indicated as follow :

$C=L(B)=B \oplus(B<<<2) \oplus(B<<<10) \oplus(B<<<18) \oplus(B<<<24)$

And $L^{\prime}($.$) indicated as follow:$

$$
C=L^{\prime}(B)=B \oplus(B<<<13) \oplus(B<<<23)
$$

\section{Applied SMS4 to encryption QR codes}

Step 1, converting the string "hellothenewworld" into plaintext QR code by using a normal QRpager gold. We regard it as a control group. as shown in the figure 2(a).

Step 2, converting the string "hellothenewworld" into cryptographic string, secret key is "hellothenewworld" too. Then using a normal QRpager gold to transform the cryptograph into QR code, as shown in the figure 2(b).

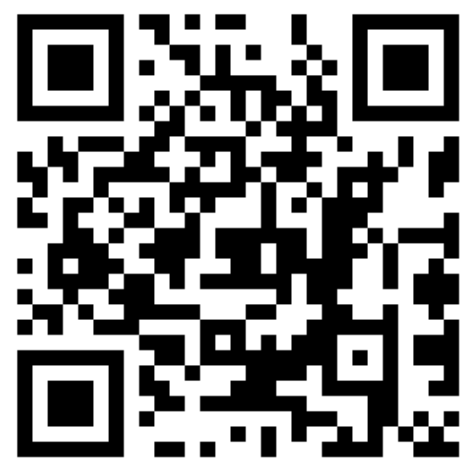

plaintext QR code

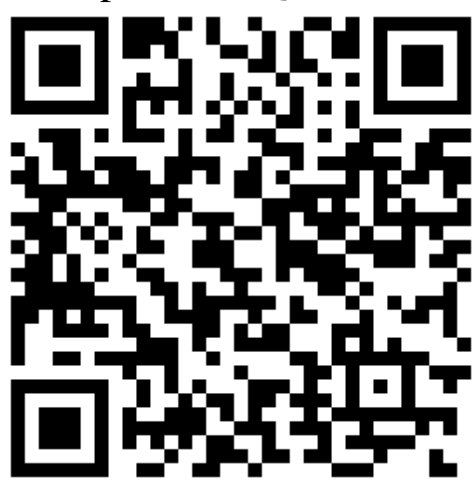

(b) encryption QR code

Figure 2 QR code 


\section{Decryption}

We will decode the two QR code obtain form above-mentioned. We get two pices of information as follow by using a mobile phone built-in decoder. It is quite clear that we can get the right information from plaintext QR code while we just get a messy code when we decode the cryptographic string.

hellothenewworld

(a) plaintext QR code

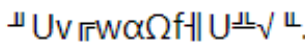

(b) encryption QR code

Figure 3 QR code

\section{References}

[1] SMS4 cipher algorithm using in Wireless local area network (LAN) products, National commercial password management office, http: //www.oscca.gov.cn/.

[2] William Stallings, network security essentials: applications and standards, 5e, PEARSON EDUCATION ASIA LIMITED and TSINGHUA UNIVERSITY PRESS, Beijing, 2013.

[3] Song Yang, Research of QR Code Encryption Method Based on Cellular Automata, MS. Harbin University of Science and Technology, March, 2014. 